\title{
Self-optimising reactive extractions: towards the efficient development of multi-step continuous flow processes
}

\author{
Adam D. Clayton ${ }^{1}$ (D) - Luke A. Power ${ }^{1}$ (D) - William R. Reynolds ${ }^{1} \cdot$ Caroline Ainsworth $^{2} \cdot$ David R. J. Hose $^{2}$ (D) \\ Martin F. Jones $^{2} \cdot$ Thomas W. Chamberlain $^{1} \cdot$ A. John Blacker ${ }^{1}$ (I) $\cdot$ Richard A. Bourne $^{1} \mathbb{B}$
}

Received: 29 November 2019 / Accepted: 30 January 2020 / Published online: 19 February 2020

(C) The Author(s) 2020

\begin{abstract}
Downstream purification of products and intermediates is essential for the development of continuous flow processes. Described herein, is a study on the use of a modular and reconfigurable continuous flow platform for the self-optimisation of reactive extractions and multi-step reaction-extraction processes. The selective extraction of one amine from a mixture of two similar amines was achieved with an optimum separation of $90 \%$, and in this case, the black-box optimisation approach was superior to global polynomial modelling. Furthermore, this methodology was utilised to simultaneously optimise the continuous flow synthesis and work-up of $N$-benzyl- $\alpha$-methylbenzylamine with respect to four variables, resulting in a significantly improved purity.
\end{abstract}

Keywords Continuous flow $\cdot$ Self-optimisation $\cdot$ Automation $\cdot$ Liquid-liquid extraction $\cdot$ Algorithm $\cdot$ Amines

\section{Introduction}

The synthesis of active pharmaceutical ingredients (APIs) requires complex multi-step processing, involving chemical transformations, reaction quenching, work-ups, extractions and purifications. Traditionally, this has been achieved by

\section{Article highlights}

- Design and implementation of a modular and reconfigurable selfoptimising continuous flow reaction and work-up system.

- Black-box optimisation favoured over polynomial modelling for selective $\mathrm{pH}$-based liquid-liquid extraction of amines.

- A simultaneous self-optimisation approach towards the efficient development of multi-step continuous flow processes.

Electronic supplementary material The online version of this article (https://doi.org/10.1007/s41981-020-00086-6) contains supplementary material, which is available to authorized users.

\section{A. John Blacker}

j.blacker@leeds.ac.uk

$\triangle$ Richard A. Bourne

r.a.bourne@leeds.ac.uk

$1 \quad$ Institute of Process Research and Development, School of Chemistry \& School of Chemical and Process Engineering, University of Leeds, Leeds LS2 9JT, UK

2 Chemical Development, Pharmaceutical Technology and Development, AstraZeneca, Macclesfield SK10 2NA, UK iterative step-by-step transformations in batch, where intermediates are purified and isolated between each synthetic step [1]. However, this process has a very high space-time demand, as large inventories of intermediates must be stored and transported between different manufacturing sites. In contrast, continuous flow offers in-line purification and the addition of reagents at set points in the processing sequence, thus providing a more productive uninterrupted reaction network [2]. Consequently, there has been a rise in the use of modular flow platforms for the multi-step synthesis of APIs, thus minimising the impact of supply chain disruptions [3-5].

The manual, iterative, optimisation of each step in a chemical processes is labour intensive, which reduces the pace of pharmaceutical development and creates a significant bottleneck for the delivery of new medicines. Self-optimisation, which combines continuous flow reactors, online analysis and optimisation algorithms, provides a rapid and autonomous approach to process optimisation [6]. This reduces the amount of time a researcher spends conducting repetitive routine experimentation, allowing them to focus on the more challenging aspects of chemical discovery and development. Many improvements have been reported in this area over the past decade, predominantly focusing on optimisation algorithms [7-9] and different analytical techniques [10-12]. However, these reports have been limited to low complexity optimisations of single step reactions. These fail to consider 
downstream unit operations, which are crucial for the purification of products and intermediates in end-to-end continuous flow synthesis.

There has been a concomitant emergence of in-line liquidliquid extraction (LLE) technologies with the advent of multistep continuous flow processes. These include both gravitybased [13] and membrane-based LLE systems [14]. Notably, a membrane-based separator was recently developed which utilises an internal diaphragm for integrated pressure control, thus providing a modular plug-and-play device [15]. This enabled the development of a reconfigurable self-optimising flow system by Jamison et al., which was subsequently used to optimise six different transformations including multi-step chemical processes [16]. However, only the reaction conditions were varied in each optimisation, despite the potential impact variables such as $\mathrm{pH}$ and solvent ratios have on the efficiency and volume productivity of downstream work-ups. Typically, reactive LLEs have been optimised using either statistical [17] or physicochemical based modelling, [18, 19] which require relatively small amounts of material. However, modelling approaches suffer from an increased complexity with an increasing number of species and/or protic sites in solution, which can result in low accuracy predictions [18].

\section{Results and discussion}

\section{Automated flow reactor}

The aim of our study was to investigate an automated blackbox optimisation approach for $\mathrm{pH}$-based liquid-liquid extraction and multi-step reaction-extraction process in continuous flow. Initially, a modular continuous flow system was constructed which could easily be reconfigured for the optimisation of either single or multi-step processes (Fig. 1). The system was comprised of three interchangeable modules: a variable temperature $\left(-40\right.$ to $\left.150{ }^{\circ} \mathrm{C}\right)$ microreactor; a miniature continuous stirred tank reactor (CSTR), fReactor, for flow rate independent mixing of liquid-liquid biphasic mixtures; [20] a membrane-based liquid-liquid separator fitted with a hydrophobic PTFE membrane $(0.5 \mu \mathrm{m})$ [15]. HPLC pumps were used for reagent addition at the inlets of the reactor and mixer, for the chemical transformation and reactive extraction steps respectively. The organic outlet stream from the separator was directed towards an on-line HPLC for analysis. Note, that there is no on-line $\mathrm{pH}$ measurement in the set-up. System parameters such as reactor temperature and pump flow rates were adjusted using a single computer terminal with integrated software control. The feedback loop was closed using the Stable Noisy Optimisation by Branch and Fit (SNOBFIT) algorithm, which is a global optimisation algorithm for bound constrained noisy optimisation of objective functions [21]. The SNOBFIT algorithm was set to conduct four experiments

per iteration, thus balancing experimental speed with optimisation efficiency.

\section{Separation of structurally similar amines}

\section{Black-box optimisation}

The presence of structurally similar impurities presents a significant challenge during multi-step continuous flow processes. These can arise as a result of incomplete reactions or limited product selectivity. The ability to control these during the reaction is not always possible, therefore rigorous optimisation of downstream purification steps is required to provide a robust process. In cases where compounds possess acidic or basic functional groups, in-line purification can be achieved via $\mathrm{pH}$-based LLE [22]. This technique is commonly used for purifying reactions involving organic amines or acids. The protonation of amines under acidic conditions reverses their solubility properties, causing them to favourably partition into the aqueous phase. This can be used to remove either unreacted amine starting materials or products from the organic reaction medium [23]. However, the pH-based LLE of mixtures containing two or more different amines presents a significantly more challenging optimisation problem.

To study an automated black-box optimisation approach for $\mathrm{pH}$-based LLE, we investigated the separation of $\alpha$ methylbenzylamine $\mathbf{1}$ and $N$-benzyl- $\alpha$-methylbenzylamine $\mathbf{2}$ in toluene (Scheme 1). The ratio of $\mathbf{1 : 2}$ was 95:5, thus representing a process in which $\mathbf{2}$ is formed as a minor impurity. The aim of the optimisation was to extract $\alpha$ methylbenzylamine 1 into the aqueous phase whilst retaining the $N$-benzyl- $\alpha$-methylbenzylamine $\mathbf{2}$ in the organic phase, as defined by Eq. (1). Thus, $\Delta$ amine $_{(\text {org })}=\%$ amine $2-\%$ amine $\mathbf{1}$, where the $\%$ amine $\mathbf{2}$ is the percentage of $\mathbf{2}$ out of the original 5\% and $\%$ amine $\mathbf{1}$ is the percentage of $\mathbf{1}$ out of the original $95 \%$. This was achieved by forming the conjugate acid 3 of $\alpha$-methylbenzylamine under acidic conditions. Three pumps were used to flow the amine mixture, nitric acid stock and diluent (water) directly into the CSTR mixing module. The inlet $\mathrm{pH}$ and solvent volume ratio $\left(V_{R}\right)$ Eq. (2) were varied by adjusting the respective pump flow rates. The optimisation boundaries were selected to provide an informative search area around the conditions required for complete protonation of the amine mixture, under the assumption of complete acid dissociation. Each experiment was run for two reactor volumes to achieve steady-state and sampled before running the next experiment.

where : subject to :

$$
\begin{aligned}
& \text { maximise }\left[\text { amine }_{(\text {org })}\right] \\
& \text { samine }_{(\text {org })}=\% \text { amine } \mathbf{2}-\% \text { amine } \mathbf{1} \\
& \text { Inlet } \mathrm{pH} \in[0.358,0.873] \\
& V_{R} \in[0.8,2.0]
\end{aligned}
$$




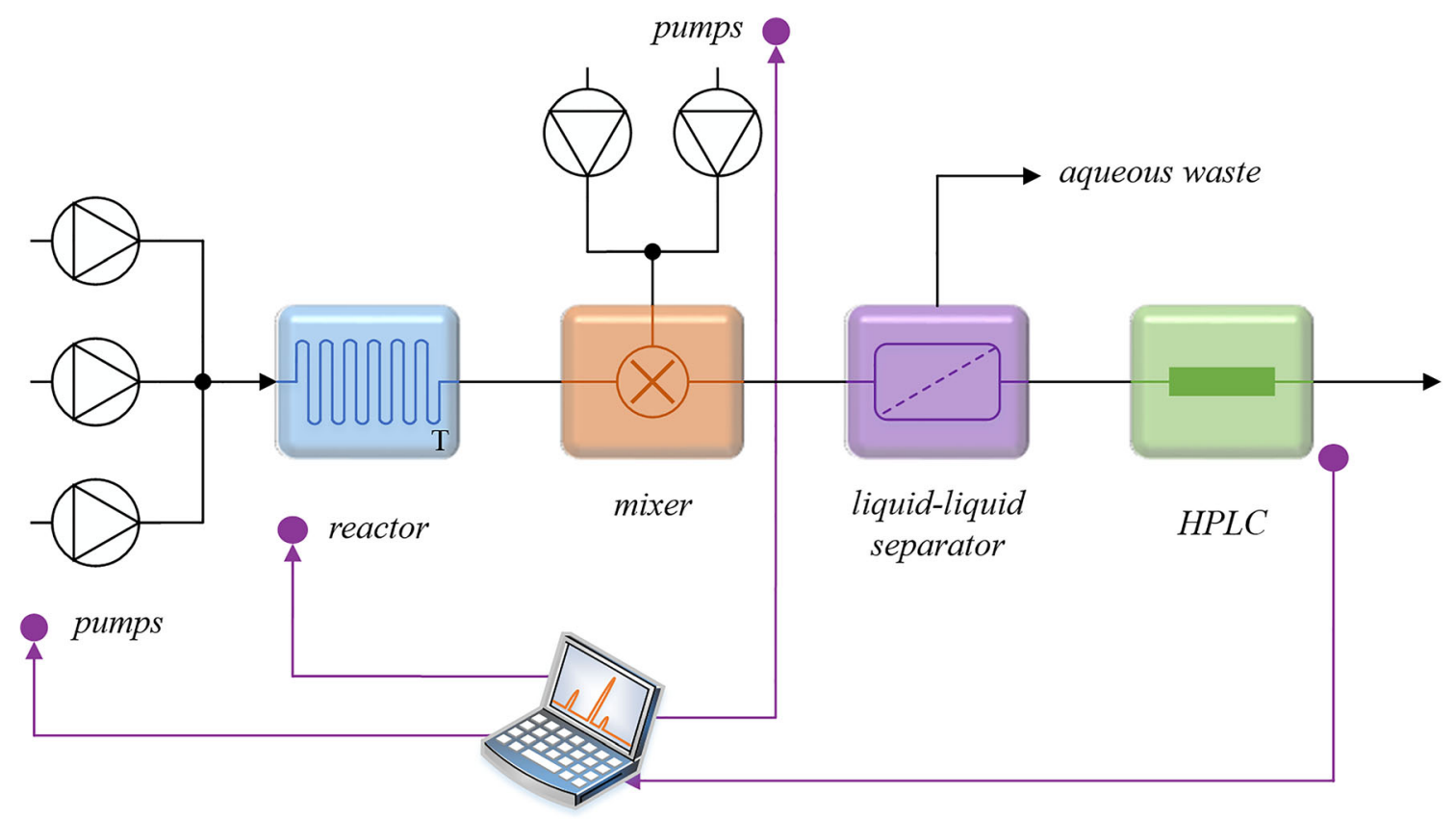

Fig. 1 Self-optimising reconfigurable continuous flow system

$V_{R}=\frac{\text { Volume }_{(\text {org })}}{\text { Volume }_{(a q)}}$

The results of the optimisation were visualised in real-time, and the optimisation manually terminated once the user was satisfied that sufficient exploration had occurred with no further improvements. In this case, the optimum was rapidly identified in just 15 experiments, corresponding to a $90 \%$ separation at a calculated inlet $\mathrm{pH}$ of 0.420 , and $V_{R}$ of 1.0 . This yielded an improvement in the purity of amine $\mathbf{1}$ from $95 \%$ in the starting mixture to $99.5 \%$ in the resultant aqueous solution under the optimised conditions. The optimisation was run for an additional 46 experiments, where exploration predominantly focused on the region around the optimum, revealing the presence of a cliff edge in the local response surface (Fig. 2b). Inspection of the individual concentration profiles for each amine in the organic phase (Fig. 2a) showed that $\alpha$-methylbenzylamine 1 was preferentially protonated over $N$ benzyl- $\alpha$-methylbenzylamine 2 in an inlet $\mathrm{pH}$ range of 0.420 0.873 and $V_{R}$ range of 1.0-2.0. Any decrease in the inlet $\mathrm{pH}$ or $V_{R}$, from the optimum conditions, resulted in a sharp decrease in the amount of $N$-benzyl- $\alpha$-methylbenzylamine 2 remaining in the organic phase, which corresponded to the observed cliff edge in the separation response surface. These results suggested that $\alpha$-methylbenzylamine 1 was more basic than $N$ benzyl- $\alpha$-methylbenzylamine 2 , which was verified by pKa determination discussed below. Furthermore, as $V_{R}$ directly affected the amount of acid available in the system, inclusion of this variable in the optimisation enabled the partitioning of the species to be finely-tuned.

Due to the presence of a sharp change in the response surface, fine-tuning of the extraction conditions was crucial for the successful optimisation of this system. Automated continuous flow platforms are well suited for this, as they provide precisely adjustable flow rates and effectively remove human error. In addition, this approach is less labour intensive compared to manual titration-based methods, requiring only $13 \mathrm{~h}$ of unsupervised experiments to identify the optimum with no prior knowledge of the system. Furthermore, the use of statistical modelling approaches, such as design of experiments, are not appropriate for global optimisation problems with sharp changes in the response surface. This is due to the poor ability

Scheme $1 \mathrm{pH}$-based liquid-liquid extraction of $\alpha$ methylbenzylamine 1 and $N$-benzyl- $\alpha$-methylbenzylamine 2

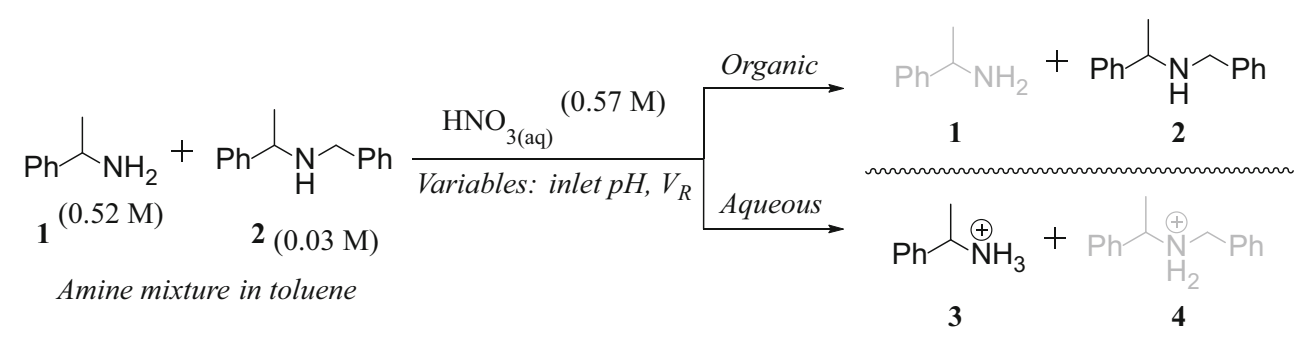



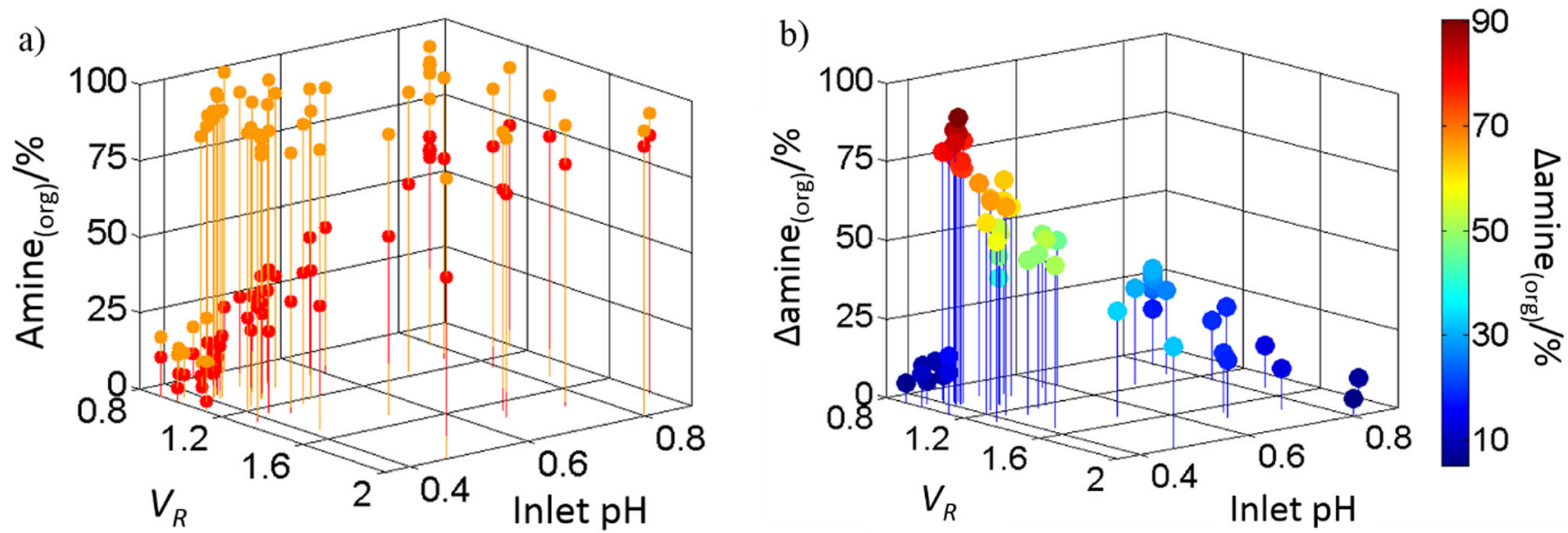

Fig. 2 Results of the self-optimising pH-based LLE: a percentage of each amine remaining in the organic phase, $\bullet=\alpha$-methylbenzylamine $\mathbf{1}$, $\bullet=N$ benzyl- $\alpha$-methylbenzylamine 2 ; b percentage difference of amines remaining in the organic phase

of polynomial models to fit sharp changes in response over a wide variable range. This is shown in Fig. 3a, where a global polynomial model of all the experimental data failed to identify the optimum, and accurately describe the true nature of the response surface around the optimum. In contrast, the SNOBFIT algorithm fits local polynomial models in subsections of the experimental space. An example of this is shown in Fig. 3b, where a local model was fit around the optimum using data in an inlet $\mathrm{pH}$ range of $0.358-0.500$ and $V_{R}$ range of $0.8-1.2$. This provided a model that successfully detected the experimentally observed cliff edge, which is information that is important for determining the region of process stability in a quality by design approach. However, quadratic polynomial models are relatively rigid designs, which in this case resulted in the incorrect prediction of equal gradients at either side of the optimum. In contrast, Gaussian processes (GPs) are significantly more versatile, providing global (Fig. 3c) and local (Fig. 3d) models which better reflected the morphology of the experimentally observed response surface, albeit at the cost of overfitting in regions of sparse data. This suggests that the use of GP based algorithms for self-optimisation would result in an overall more efficient optimisation. However, different GP model parameters and hyperparameters can result in significantly different models, and therefore rigorous optimisation of these settings is required to obtain reproducible predictions.

\section{pKa determination}

The $\mathrm{pKa}$ of the conjugate acid $(\mathrm{pKaH})$ for each amine was determined via titration with hydrochloric acid in triplicate using the "half-volume" method. The "half-volume" refers to the volume of acid added equal to half that required to reach the equivalence point, where $\left[\mathrm{A}^{-}\right]=[\mathrm{HA}]$ and $\mathrm{pKa}=\mathrm{pH}[24]$. The resultant titration and first derivative plots are shown in Fig. 4. The volume added at the half-height of the peak (i.e. largest change in $\mathrm{pH}$ per change in volume) was correlated with the titration curves to determine the $\mathrm{pKaH}$. The $\mathrm{pKaH}$ of $\alpha$-methylbenzylamine $\mathbf{1}$ and $N$-benzyl- $\alpha$-methylbenzylamine $\mathbf{2}$ were characterised as $9.33 \pm 0.02$ and $7.77 \pm 0.02$ respectively. These were found to be in good agreement with Conductor like Screening Model for Real Solvents (COSMO-RS) predictions of 9.32 for $\alpha$ methylbenzylamine $\mathbf{1}$ and 7.73 for $N$-benzyl- $\alpha$ methylbenzylamine 2 [25]. This data supports the previous observation that $\alpha$-methylbenzylamine $\mathbf{1}$ is more basic than $N$ benzyl- $\alpha$-methylbenzylamine 2 .

\section{Tandem reaction-extraction optimisation}

One of the main challenges associated with continuous multi-step process development is the identification of reaction and downstream work-up conditions that are complimentary, without any a priori knowledge of how the sequential steps are related. With a suitable system for the automated optimisation of LLEs in hand, our attention shifted to the simultaneous optimisation of a tandem reaction and extraction process. For this, the synthesis and purification of $N$-benzyl- $\alpha$-methylbenzylamine $\mathbf{2}$ in continuous flow was investigated (Scheme 2) [7]. As previously, aqueous nitric acid was introduced into the CSTR mixing module for the removal of amine-containing impurities. The reaction was optimised with respect to residence time and temperature, and the LLE was optimised with respect to inlet $\mathrm{pH}$ and $V_{R}$. The boundary conditions for the optimisation of the reaction and extraction were selected based on knowledge of the system gained from previous work and this work respectively [7]. The aim of the optimisation was to maximise the purity of $N$-benzyl- $\alpha$ methylbenzylamine $\mathbf{2}$ with respect to the following impurities: (i) unreacted $\alpha$-methylbenzylamine 1; (ii) unreacted benzyl bromide 5; (iii) tertiary amine by-product 6; (iv) conjugate acid of diisopropylethylamine (DIPEA) 7. The absolute purity of $N$-benzyl- $\alpha$-methylbenzylamine $\mathbf{2}$ in the 
Fig. 3 Contour plots showing statistical models derived from the self-optimisation data: a global polynomial model from all data, dashed boxed highlights local area around the optimum; $\mathbf{b}$ local polynomial model exclusively from data around the optimum; c global GP model from all data, dashed boxed highlights local area around the optimum, contour labels omitted for clarity; d local GP model exclusively from data around the optimum, contour labels omitted for clarity. $\star=$ experimental optimum with model predictions a) Global Polynomial Model

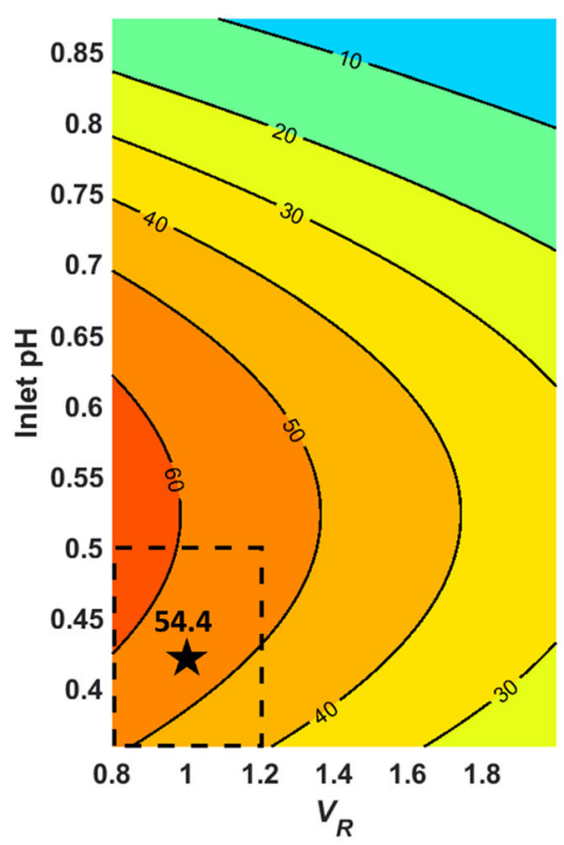

c) Global GP Model

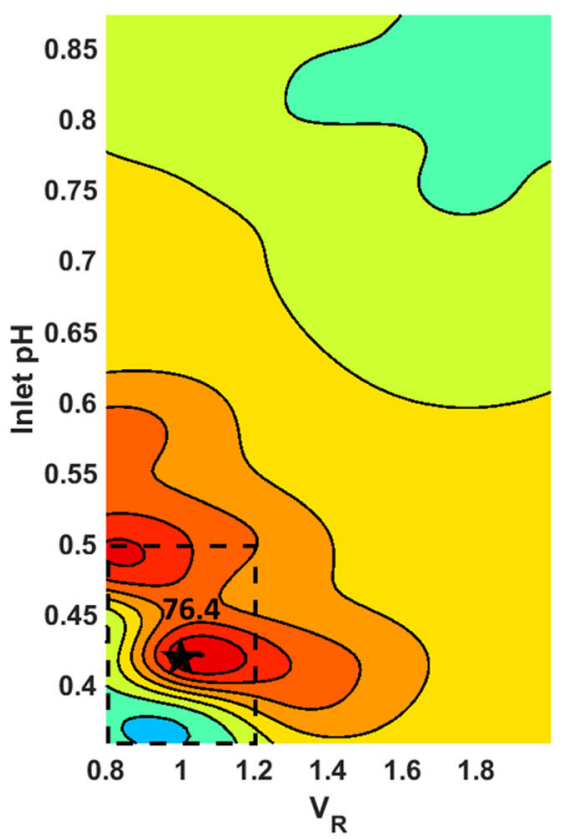

b) Local Polynomial Model

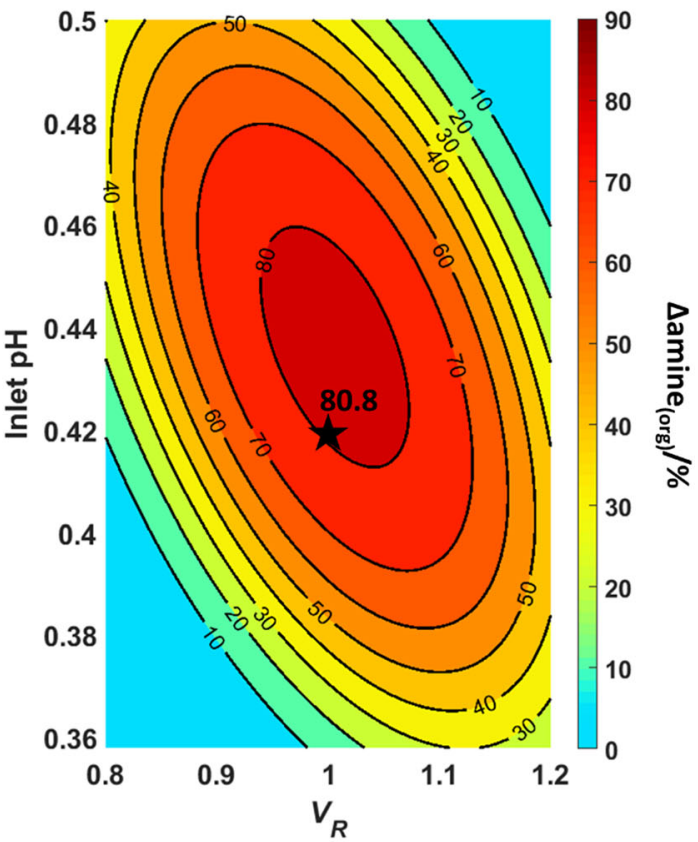

d) Local GP Model

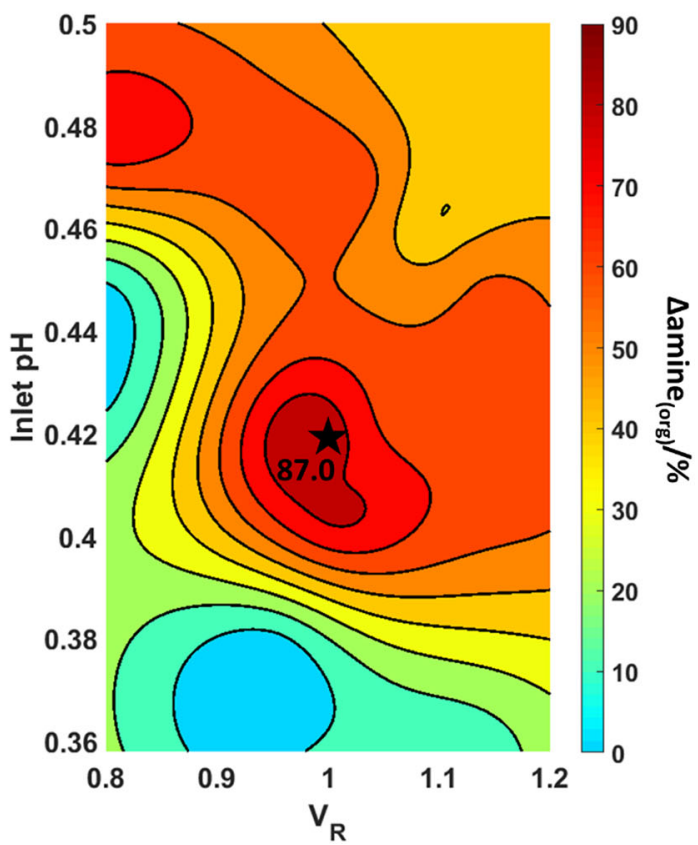

organic phase was measured via on-line HPLC. Using purity as the objective function ensured that the optimisation would favour a high yielding reaction step and efficient extraction conditions, by minimising the amount of unreacted benzyl bromide and amine-containing impurities respectively. The SNOBFIT algorithm was selected for this system, owing to its success in optimising the LLE in the previous example.

The results of the optimisation are shown in Fig. 5. An optimum purity of $71 \%$ at a yield of $63 \%$ was identified at the following process conditions: $\mathrm{t}_{\mathrm{res}}=6.9 \mathrm{~min}$, temperature $=$ $127.2{ }^{\circ} \mathrm{C}$, inlet $\mathrm{pH}=0.772$ and $V_{R}=2.45$. In terms of reaction conditions, a high purity was favoured at high temperatures and short residence times. High temperatures were found to drive the reaction to high conversions, where the reduction in unreacted starting materials outweighed the increase in the formation of tertiary amine $\mathbf{6}$ with respect to the purity of $N$ benzyl- $\alpha$-methylbenzylamine $\mathbf{2}$ (see ESI for impurity plots). Although the highest conversions were observed at longer residence times, this corresponded to an increase in the 

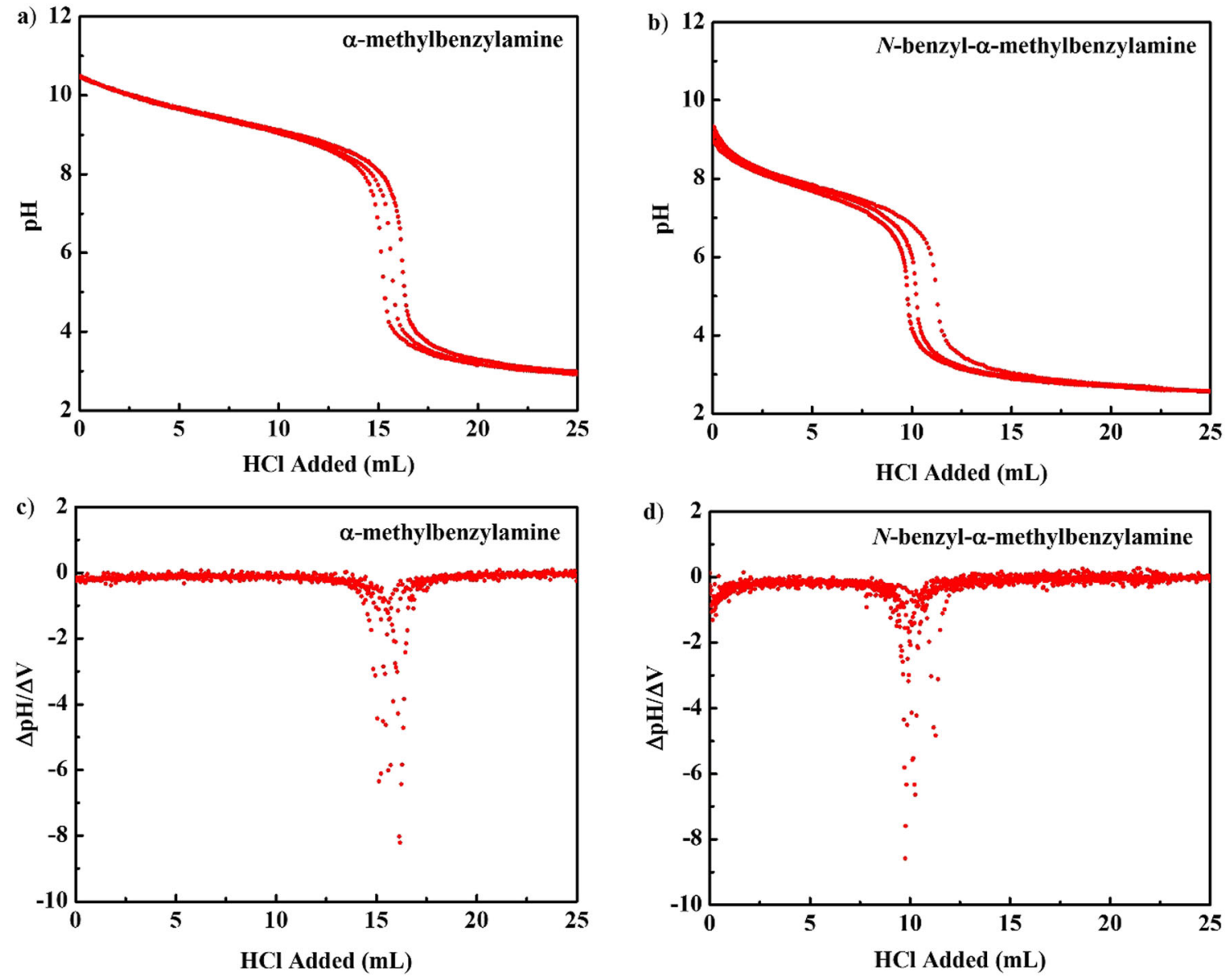

Fig. 4 Titration curves and first derivative plots carried out in triplicate: a titration curve for $\alpha$-methylbenzylamine 1 ; $\mathbf{b}$ titration curve for $N$-benzyl- $\alpha$ methylbenzylamine 2; $\mathbf{c}$ first derivative plot for $\alpha$-methylbenzylamine $\mathbf{1}$; d first derivative plot for $N$-benzyl- $\alpha$-methylbenzylamine 2

concentration of salt 7 , which was not efficiently extracted from the organic phase in this region. Similar to the previous LLE example, there was a noticeable cliff edge around the optimum, where decreasing $V_{R}$ from 2.45 to 1.32 corresponded to a decrease in purity from $71 \%$ to $16 \%$. This could mainly be attributed to the salt 7 impurity, which disfavoured extraction from the organic phase when $V_{R}$ was less than 1.5. Nevertheless, a comparison of the optimum reaction conditions, including and excluding the downstream LLE module, showed that the optimised extraction significantly improved the purity of $N$-benzyl- $\alpha$-methylbenzylamine 2 from $38 \%$ to $71 \%$. The aqueous acidic work-up reduced the
Scheme 2 Optimisation parameters for the continuous flow synthesis of $N$-benzyl- $\alpha$ methylbenzylamine 2 via $N$ benzylation of $\alpha$ methylbenzylamine $\mathbf{1}$, and subsequent downstream work-up of the crude reaction mixture

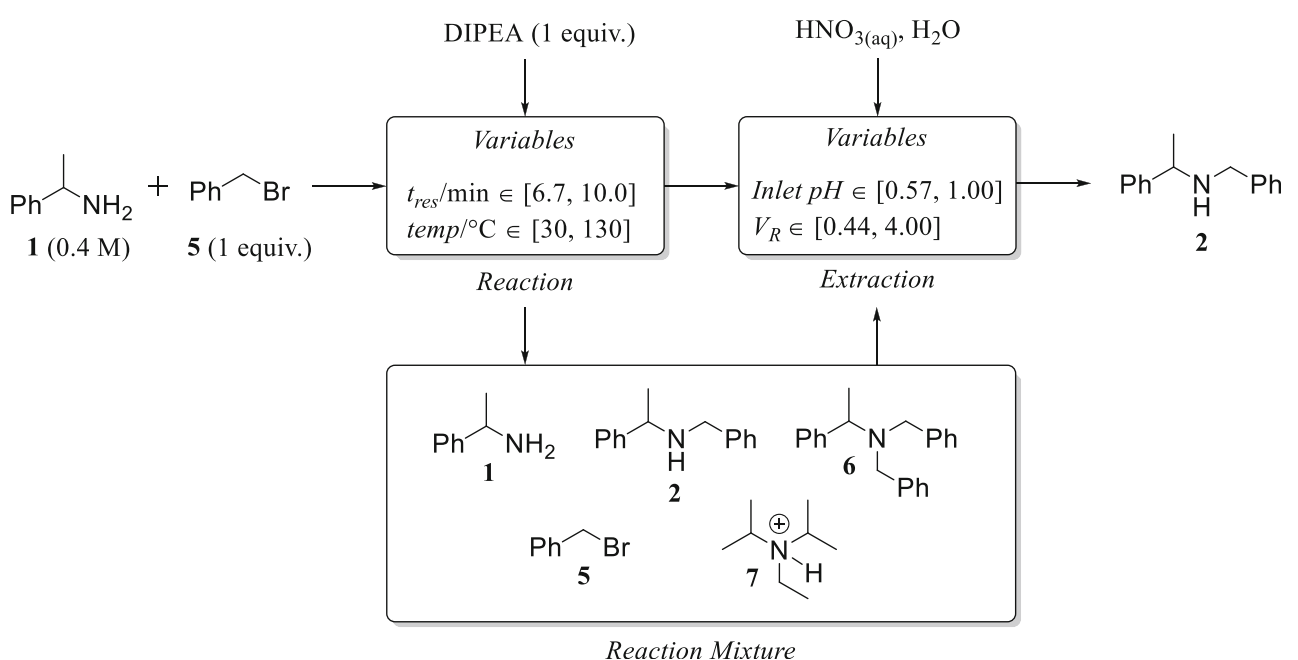


Fig. 5 Self-optimisation results for the continuous flow synthesis and work-up of $N$-benzyl- $\alpha$ methylbenzylamine 2 with respect to purity. $\downarrow$ maximum purity

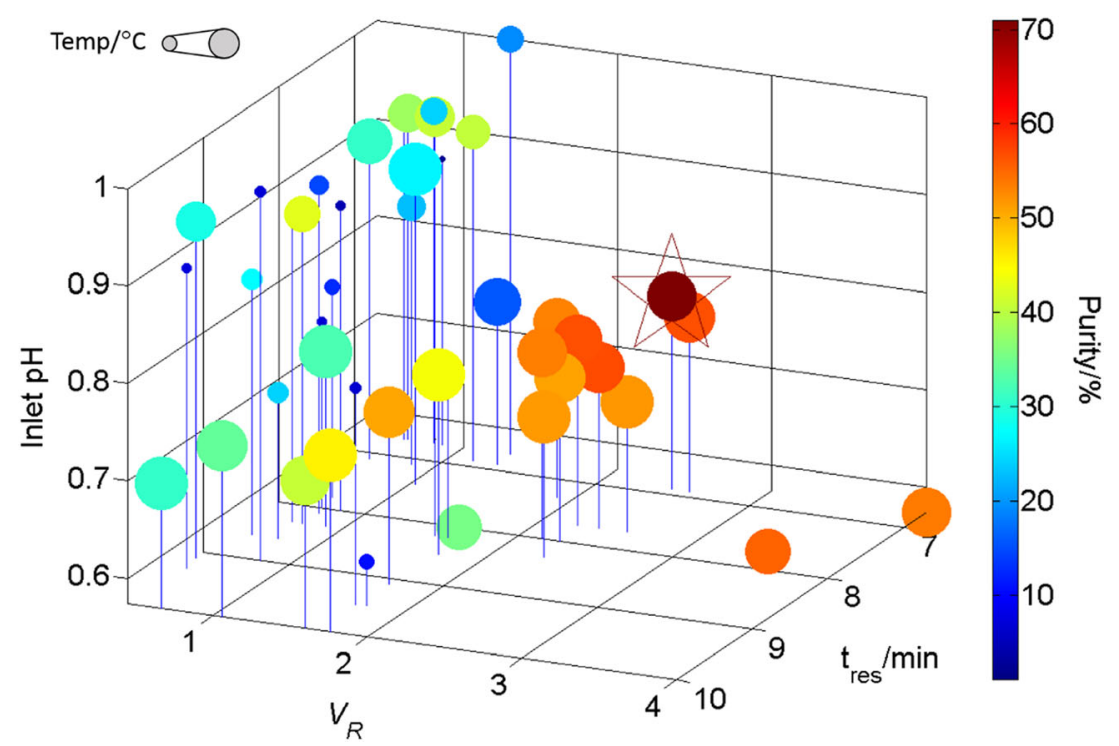

amount of salt 7 by $81 \%$, whilst selectively extracting $43 \%$ of the unreacted $\alpha$-methylbenzylamine 1 starting material. The purity might be improved further using a multi-stage LLE, which was beyond the scope of this study, and is the subject of current work [26].

\section{Conclusions}

We have successfully developed a modular and reconfigurable continuous flow platform for the self-optimisation of multistep reaction and extraction processes. A detailed SNOBFIT optimisation for the selective $\mathrm{pH}$-based extraction of an amine mixture was conducted. Optimum conditions for the separation of $\alpha$-methylbenzylamine 1 and $N$-benzyl- $\alpha$ methylbenzylamine 2 were found to be an inlet $\mathrm{pH}$ of 0.420 and $V_{R}$ of 1.0 , providing a $90 \%$ separation. The optimum was identified in just 15 experiments, and a total of 61 unsupervised experiments were conducted in $13 \mathrm{~h}$, revealing a cliff edge in the local response surface around the optimum. Notably, the use of an automated black-box optimisation approach overcame the challenges associated with labour intensive manual experimentation and low accuracy polynomial modelling. Furthermore, inclusion and optimisation of $V_{R}$ as a variable, which is uncommon during process development, is instructive for the optimisation of work-ups, having an impact upon efficiency and volume productivity. With the increase in multi-step continuous flow processes for the synthesis of APIs, we investigated the optimisation of a tandem reaction-extraction process. By applying the same black-box optimisation methodology developed for LLEs, we were able to simultaneously optimise the continuous flow synthesis and purification of $N$-benzyl- $\alpha$-methylbenzylamine 2 with respect to four variables in just 53 experiments with no human intervention. A purity of $71 \%$ was achieved at a residence time of 6.9 min, temperature of $127.2^{\circ} \mathrm{C}$, inlet $\mathrm{pH}$ of 0.772 and $V_{R}$ of 2.45. Inclusion of the LLE in the optimisation resulted in an increase in purity from $38 \%$ to $71 \%$, by selective removal of salt by-products and unreacted amine starting materials. This work demonstrated that the efficient optimisation of reactive extractions and multi-step continuous flow processes can be achieved using self-optimisation technology, and highlighted that future improvements could be made by incorporating GP based algorithms. Furthermore, we envisage that by considering downstream unit operations during initial reaction optimisation, scaling transitions can be significantly simplified.

Acknowledgements ADC (EP/N509681/1) and LAP (EP/S513829/1) thank the EPSRC, University of Leeds and AstraZeneca for CASE student funding.

Open Access This article is licensed under a Creative Commons Attribution 4.0 International License, which permits use, sharing, adaptation, distribution and reproduction in any medium or format, as long as you give appropriate credit to the original author(s) and the source, provide a link to the Creative Commons licence, and indicate if changes were made. The images or other third party material in this article are included in the article's Creative Commons licence, unless indicated otherwise in a credit line to the material. If material is not included in the article's Creative Commons licence and your intended use is not permitted by statutory regulation or exceeds the permitted use, you will need to obtain permission directly from the copyright holder. To view a copy of this licence, visit http://creativecommons.org/licenses/by/4.0/.

\section{References}

1. Webb D, Jamison TF (2010) Continuous flow multi-step organic synthesis. Chem Sci 1:675-680

2. Britton J, Raston CL (2017) Multi-step continuous-flow synthesis. Chem Soc Rev 46:1250-1271 
3. Adamo A, Beingessner RL, Behnam M, Chen J, Jamison TF, Jensen KF, Monbaliu J-CM, Myerson AS, Revalor EM, Snead DR, Stelzer T, Weeranoppanant N, Wong SY, Zhang P (2016) On-demand continuous-flow production of pharmaceuticals in a compact, reconfigureable system. Science 352:61-67

4. Baumann M, Baxendale IR (2015) The synthesis of active pharmaceutical ingredients (APIs) using continuous flow chemistry. Beilstein J Org Chem 11:1194-1219

5. Snead DR, Jamison TF (2015) A three-minute synthesis and purification of ibuprofen: pushing the limits of continuous-flow processing. Angew Chem Int Ed 54:983-987

6. Clayton AD, Manson JA, Taylor CJ, Chamberlain TW, Taylor BA, Clemens G, Bourne RA (2019) Algorithms for the self-optimisation of chemical reactions. React Chem Eng 4:1545-1554

7. Schweidtmann AM, Clayton AD, Holmes N, Bradford E, Bourne RA, Lapkin AA (2018) Machine learning meets continuous flow chemistry: automated optimization towards the Pareto front of multiple objectives. Chem Eng J 352:277-282

8. Houben C, Peremezhney N, Zubov A, Kosek J, Lapkin AA (2015) Closed-loop multitarget optimization for discovery of new emulsion polymerization recipes. Org Process Res Dev 19:1049-1053

9. Cortés-Borda D, Kutonova KV, Jamet C, Trusova ME, Zammattio F, Truchet C, Rodriguez-Zubiri M, Felpin F-X (2016) Optimizing the heck-Matsuda reaction in flow with a constraint-adapted direct search algorithm. Org Process Res Dev 20:1979-1987

10. Holmes N, Akien GR, Savage RJD, Stanetty C, Baxendale IR, Blacker AJ, Taylor BA, Woodward RL, Meadows RE, Bourne RA (2016) Online quantitative mass spectrometry for the rapid adaptive optimisation of automated flow reactors. React Chem Eng 1:96-100

11. Sans V, Porwol L, Dragone V, Cronin L (2015) A self optimizing synthetic organic reactor system using real-time in-line NMR spectroscopy. Chem Sci 6:1258-1264

12. Skilton RA, Parrott AJ, George MW, Poliakoff M, Bourne RA (2013) Real-time feedback control using online attenuated total reflection Fourier transform infrared (ATR FT-IR) spectroscopy for continuous flow optimization and process knowledge. Appl Spectrosc 67:1127-1131

13. O'Brien M, Koos P, Browne DL, Ley SV (2012) A prototype continuous-flow liquid-liquid extraction system using open-source technology. Org Biomol Chem 10:7031-7036

14. Cervera-Padrell AE, Morthensen ST, Lewandowski DJ, Skovby T, Kiil S, Gernaey KV (2012) Continuous hydrolysis and liquid-liquid phase separation of an active pharmaceutical ingredient intermediate using a miniscale hydrophobic membrane separator. Org Process Res Dev 16:888-900

15. Adamo A, Heider PL, Weeranoppanant N, Jensen KF (2013) Membrane-based, liquid-liquid separator with integrated pressure control. Ind Eng Chem Res 52:10802-10808

16. Bédard A-C, Adamo A, Aroh KC, Russell MG, Bedermann AA, Torosian J, Yue B, Jensen KF, Jamison TF (2018) Reconfigureable system for automated optimization of diverse chemical reactions. Science 361:1220-1225

17. Rewatkar K, Shende DZ, Wasewar KL (2017) Modeling and optimization of reactive extraction of Gallic acid using RSM. Chem Eng Commun 204:522-528

18. Ashworth IW, Meadows RE (2018) A general liquid-liquid partitoning equation and its consequences: learning from the $\mathrm{pH}$ dependent extraction of a pharmaceutical intermediate. J Org Chem 83:4270-4274

19. Thakre N, Prajapati AK, Mahapatra SP, Kumar A, Khapre A, Pal D (2016) Modeling and optimization of reactive extraction of citric acid. J Chem Eng Data 61:2614-2623

20. Chapman MR, Kwan MHT, King G, Jolley KE, Hussain M, Hussain S, Salama IE, Niño CG, Thompson LA, Bayana ME, Clayton AD, Nguyen BN, Turner NJ, Kapur N, Blacker AJ
(2017) Simple and versatile laboratory scale CSTR for multiphasic continuous-flow chemistry and long residence times. Org Process Res Dev 21:1294-1301

21. Huyer W, Neumaier A (2008) SNOBFIT - Stable Noisy Optimization by Branch and Fit. ACM Trans. Math. Softw 35:9 $1-25$

22. Dai C, Snead DR, Zhang P, Jamison TF (2015) Continuous-flow synthesis and purification of atropine with sequential in-line separations of structurally similar impurities. J Flow Chem 5:133-138

23. Peng SX, Henson C, Strojnowski MJ, Golebiowski A, Klopfenstein SR (2000) Automated high-throughput liquid-liquid extraction for initial purification of combinatorial libraries. Anal Chem 72:261266

24. Stephens SJ, Joncich MJ (1977) Determination of pKa using the half-volume method: a laboratory experiment. J Chem Educ 54:711

25. Software for Chemistry \& Materials, COSMO-RS. https://www. scm.com/product/cosmo-rs/. Accessed 27 Sept 2019

26. Weeranoppanant N, Adamo A, Saparbaiuly G, Rose E, Fleury C, Schenkel B, Jensen KF (2017) Design of multistage counter-current liquid-liquid extraction for small-scale applications. Ind Eng Chem Res 56:4095-4103

Publisher's note Springer Nature remains neutral with regard to jurisdictional claims in published maps and institutional affiliations.

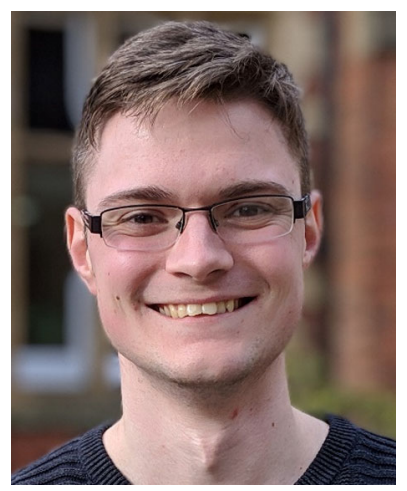

Adam Clayton received a degree in Chemistry (MChem) in 2016 from the University of Huddersfield. He then moved to the University of Leeds with an AstraZeneca CASE studentship, where he is currently studying for a PhD in Chemical and Process Engineering under the supervision of Dr Richard Bourne. Adam will be continuing as a $\mathrm{Dr}$ Reddy's funded postdoctoral researcher in the Institute of Process Research and Development from 2020. His research interests focus on the development of automated continuous flow systems for improved process design and optimisation.

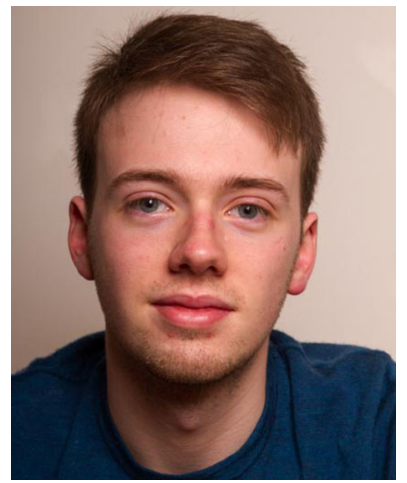

Luke Power received a degree in Natural Sciences (B.A.) in 2016 from Trinity College Dublin. He then moved to the University of Leeds to gain a degree in Chemical Engineering (M.Sc.). $\mathrm{He}$ has remained in Leeds and is currently undertaking a $\mathrm{PhD}$ with an AstraZenica CASE studentship in the school of Chemistry under the supervision of Prof. John Blacker. 